EPJ manuscript No.

(will be inserted by the editor)

\title{
Melting of a Wigner Crystal in an Ionic Dielectric
}

\author{
S. Fratini and P. Quémerais \\ Laboratoire d'Etudes des Propriétés Electroniques des Solides \\ CNRS, BP 166, 38042 Grenoble Cedex 9, France \\ Received: date / Revised version: date
}

\begin{abstract}
The melting of a Wigner Crystal of electrons placed into a host polar material is examined as a function of the density and the temperature. When the coupling to the longitudinal optical modes of the host medium is turned on, the WC is progressively transformed into a polaronic Wigner Crystal. We estimate the critical density for crystal melting at zero temperature using the Lindeman criterion. We show that above a certain critical value of the Fröhlich electron-phonon coupling, the melting towards a quantum liquid of polarons is not possible, and the insulator-to-metal transition is driven by the ionization of the polarons (polaron dissociation). The phase diagram at finite temperature is obtained by making use of the same Lindeman criterion. Results are also provided in the case of an anisotropic electron band mass, showing that the scenario of polaron dissociation can be relevant in anisotropic compounds such as the superconducting cuprates at rather moderate e-ph couplings.
\end{abstract}

PACS. PACS-key describing text of that key - PACS-key describing text of that key

\section{Introduction}

As it is well-known, the formation of a single polaron is already a many-body problem, since it deals with the interaction of one electron with an infinite number of phonons. It is thus clear that treating a system of polarons at finite density is a very complicated task. Some theories have been proposed so far which neglect the Coulomb interactions, or treat them in the random phase approximation. The very existence of polaronic bands [1] lies on this theoretical basis, although, to our knowledge, no rigorous justification of this approach has ever been given in the literature. Old but reliable results were obtained by Mahan for low electron-phonon (e-ph) coupling and high electron density [2], and extended later to intermediate eph coupling by Lemmens et al. [3]. Recently, De Filippis et al. [1] obtained interesting results valid at lower densities and higher e-ph coupling, giving a more complete physical insight to the problem in the metallic phase.

A completely different approach of the same problem can be given starting from the low density regime, where the particles localize due to the long-range Coulomb repulsion [5, 6, 7]. In this limit, the treatment is somehow simplified because the statistical effects between the electrons can be neglected. The simplest way to introduce the problem we are dealing with is to consider a Wigner Crystal (WC) of electrons, which is the ground-state of the electron gas at low density, and dip it into a polar (ionic) host material. What are the effects of the polarization on the stability of the WC?

Send offprint requests to: quemerai@lepes.polycnrs-gre.fr
A polar dielectric is a compound which is characterized by two main sources of polarization: the electronic polarization, corresponding to the vibrations of the core electrons at optical frequencies $\left(10^{14-15} s^{-1}\right)$, and the ionic polarization, which is carried by the longitudinal optical modes in the infra-red region $\left(\omega_{L O} \approx 10^{13} \mathrm{~s}^{-1}\right)$, corresponding to the relative motion of the positive and negative ions. An external charge moving in such material [8] interacts with the dipoles of the dielectric, and produces a polarization cloud which partially screens its Coulomb field. The composite state of an electron plus its induced polarization is called a polaron [9]. Such a quasi-particle has a finite extension (the polaron radius), a finite selfenergy (the energy required to form the bound state), and a renormalized mass $M_{P}$ which is generally greater than the electron band mass. As customary and following Frohlich [10], we can define a dimensionless e-ph coupling as $\alpha=\left(m^{*} / 2 \hbar^{3} \omega_{L O}\right)^{1 / 2} e^{2} / \tilde{\varepsilon}$, which is the only relevant parameter for a single isolated polaron. Here $m^{*}$ is the electron band mass and the effective dielectric constant $\tilde{\varepsilon}$ is defined as $1 / \tilde{\varepsilon}=1 / \varepsilon_{\infty}-1 / \varepsilon_{s}$, where $\varepsilon_{s}$ and $\varepsilon_{\infty}$ are respectively the static and high frequency dielectric constants of the medium.

If we now consider two interacting polarons, two different situations can occur. Under some very specific conditions, the two particles can attract and form a bound state, sharing the same polarization cloud. The properties of such composite bosons - the bipolarons - have been extensively studied, especially in connection to their Bose condensation at low enough temperatures. However, bipolarons can only exist if the ratio $\eta=\varepsilon_{\infty} / \varepsilon_{s}$ is lower 
S. Fratini, P. Quémerais: Melting of a Wigner Crystal in an Ionic Dielectric

than a critical value $\eta_{c} \approx 0.1$, and for rather large e-ph couplings $(\alpha>6-7)\left[11\right.$. On the other hand, for $\eta>\eta_{c}$, which is quite common in polar materials, two polarons repel at large distances as $1 / \varepsilon_{s} d$. Throughout the present paper, we will be dealing with compounds where the net interaction between polarons is repulsive.

As was stated previously, a reliable treatment of the many-polaron problem can be given starting at low density, if we assume that the particles localize due to the long-range Coulomb interactions, forming a Polaronic Wigner Crystal (PWC). In that case, the polarization of the host material will react to screen the external charges, and this will change both their interaction and kinetic energy, as compared to the ordinary WC of electrons. On one hand, the total Coulomb interaction energy is shifted from $\sim e^{2} / R_{s}$ to $\sim e^{2} / \varepsilon_{s} R_{s}, R_{s}$ being the mean distance between two electrons at a given density. On the other hand, the kinetic energy is also reduced owing to the polaron mass, and behaves as $\sim \hbar^{2} / M_{p} R_{s}^{2}$. From this simple argument, we see that the two effects above compete regarding the WC stability. While the decrease of the interaction energy favours delocalization, the decrease of the kinetic energy tends to stabilize the crystallized state.

The correct evaluation of the balance between these two effects is thus essential in order to understand the melting mechanism occurring for increasing density. It is particularly instructive to consider the adiabatic limit obtained for vanishing phonon frequency $\omega_{L O}(\alpha \rightarrow \infty)$. In that case, as given by standard polaron theory $[12$, while the polaron radius and energy remain finite, the polaron mass becomes infinite, and there is no way to get mobile polarons. Therefore, at zero temperature, the PWC can only melt through ionization of the electrons from their polarization potential-wells. The latter must be screened out by the liberated carriers in the same fashion as for the usual Mott transition in non-polar semiconductors, and the system will eventually become metallic [13. We have already published a phenomenological Mott-like criterion which describes the insulator-to-metal transition (IMT) in this situation [14]:

$$
n_{c}^{1 / 3}\left(\tilde{\varepsilon} / \varepsilon_{\infty}\right) R_{P} \approx 0.25
$$

where $R_{P}$ is the polaron radius (the bound state radius), which is the relevant localization length in our problem.

If one restores a finite phonon frequency $\left(\omega_{L O} \neq 0\right)$ as is the case in real materials, the problem becomes more intricate, since the polarons are a priori mobile (they have a finite mass). Upon increasing the density, the polarons themselves could be delocalized without being ionized, possibly leading to a polaron liquid beyond the transition. The aim of the present work is to examine carefully the quantum melting of a PWC in all the e-ph coupling regimes, as was previously done for the more simple case of the electron crystal (which is recovered for $\alpha=0$ ). Some important results have already been published in several letters 66,14, 15]. We provide here the details concerning the model, approximations and calculations, and give a more complete description including results at finite temperatures and for anisotropic compounds [16].
The paper is organized as follows. In section 2, we derive a model for the insulating state which includes both electron-phonon and electron-electron interactions, and point out the different approximations involved. Starting from the Fröhlich model, we assume a crystallized state for the polarons, and expand the long-range Coulomb interactions between different particles up to second order in the small displacements relative to their equilibrium positions, neglecting anharmonicity and exchange effects. It can be demonstrated [15,16 that the resulting dipoledipole interactions between different electrons are responsible for the dispersion of the collective modes of the PWC. Furthermore, above a certain critical density, the dipolar terms can lead to a phonon instability of the polaron lattice, corresponding to the spontaneous excitation of longwavelength transverse modes. This phenomenon, as well as its consequences on the properties of the dielectric constant of the system, will be extensively analysed in a forthcoming paper [17].

In the present work, we will neglect such non-local interaction terms, restricting ourselves to the mean-field Wigner approximation. This corresponds to including the many-body effects through an effective local potential acting on each particle. Hence, the many-polaron problem is reduced to the problem of a single polaron in an external potential. Taking advantage of this approximation, in section 3 we study the PWC in the framework of Feynman's path-integral method [18], which is known to give reliable analytical results at any values of the e-ph coupling.

In section 4 , we examine the insulator-to-metal transition occurring for increasing density (at zero temperature) in terms of the Lindemann criterion, as was done by Nozières and Pines for the electron crystal [19]. Since the polaron is a composite particle, we argue that there are two different Lindemann criteria to be considered: one for the center-of-mass motion, which describes the melting as in the ordinary $\mathrm{WC}$, and one for the relative displacement between the electron and its polarization cloud, which describes the ionization of the polarons. This allows us to distinguish between two differernt melting mechanisms, depending on the e-ph coupling strength. At weak or moderate couplings $\left(\alpha<\alpha^{*}\right)$, the situation is very much the same as in the bare electron crystal, i.e. the melting is driven by the increasing fluctuations of the localized particles. On the other hand, at strong e-ph coupling $\left(\alpha>\alpha^{*}\right)$, i.e. at low but finite $\omega_{L O}$, the crystal melting is due to the dissociation of the polarons. In that case, the electrons cannot carry with them their phonon clouds in the metallic phase, and the melting towards a degenerate polaron liquid can be excluded. We next extend the results to finite temperatures, and derive an approximate $T$ vs. $n$ phase diagram for the many-polaron problem. For an application to the superconducting cuprates, we finally generalize our treatment to systems where the electron band mass is an anisotropic quantity. The results presented in Table 1 suggest that polaron dissociation could be a real physical possibility in such compounds. 


\section{Model and approximations}

In this section, we derive our basic model for the polaron crystal. Let us consider a polar material, characterized by the three parameters aforementioned: $\omega_{L O}, \varepsilon_{s}$ and $\varepsilon_{\infty}$. We next consider an ideal doping procedure, which consists in introducing into this "host" material a finite density of electrons, which we express as $n=\left(4 \pi R_{s}^{3} / 3\right)^{-1}$, plus a rigid jellium which exactly compensates the negative charges. Owing to its polarizability, the host crystal responds to these excess charges. If we neglect magnetic effects, the total interaction energy is given by standard laws of electrostatics as 12]:

$$
\mathcal{E}_{I}=\frac{1}{4 \pi} \iint \mathbf{E} \cdot \dot{\mathbf{D}} d^{3} r d t
$$

where time integrations must be carried out along a path satisfying the equations of motion. If we suppose that the localization length scales of our problem are larger than the anion-cation distance in the host material, the latter can be treated as a continuous medium, and we can consider the total electric field $\mathbf{E}(\mathbf{r})$ and the electric displacement $\mathbf{D}(\mathbf{r})$ due to the doping charges (electrons plus jellium) as macroscopic fields. Such a treatment is standard in polaron theory, and leads to the well-known LandauFröhlich model [9, 10]. The two quantities above defined are related to the polarization field $\mathbf{P}$ through the relation $\mathbf{D}=\mathbf{E}+4 \pi \mathbf{P}$, which can be used to eliminate $\mathbf{E}$, and derive a Lagrangian which only depends on the excess charges. Following Fröhlich, we separate two sources of polarization: $\mathbf{P}=\mathbf{P}_{0}+\mathbf{P}_{i r}$. $\mathbf{P}_{0}$ corresponds to high frequency oscillating charges and can be included through the optical dielectric constant $\varepsilon_{\infty} . \mathbf{P}_{i r}$ is due to the ionic distortion, and vibrates at a frequency $\omega_{L O}$ in the infra-red range. One obtains the following interaction Lagrangian (see ref. 12] for more details about this derivation):

$L_{I}=-\frac{1}{8 \pi \varepsilon_{\infty}} \int \mathbf{D}^{2}+\int \mathbf{P}_{i r} \cdot \mathbf{D}+\frac{2 \pi \tilde{\varepsilon}}{\omega_{L O}^{2}} \int\left({\dot{P_{i r}}}^{2}-\omega_{L O}^{2} P_{i r}^{2}\right)$

where $\mathbf{P}_{i r}$ and $\left(4 \pi \tilde{\varepsilon} / \omega_{L O}^{2}\right) \dot{\mathbf{P}}_{i r}$ are conjugate moments, and integrations run over the entire space $\left(d^{3} \mathbf{r}\right.$ in the integrands has been omitted). The different terms in eq. (3) represent respectively the interactions between the excess charges, between the charges and the longitudinal optical phonons, and the free-phonon Lagrangian. We can now specify the nature of the doping charges - say electrons and positive jellium, but the same applies to holes and negative jellium - by writing $\mathbf{D}=\mathbf{D}^{-}+\mathbf{D}^{+}$, where

$$
\mathbf{D}^{-}=\sum_{i} \mathbf{D}_{i}^{-}=-e \sum_{i} \frac{\mathbf{r}-\mathbf{r}_{i}}{\left|\mathbf{r}-\mathbf{r}_{i}\right|^{3}}
$$

$\mathbf{r}_{i}$ being the electron coordinate. We will come back later on the precise formulation of the term $\mathbf{D}^{+}$due to the rigid jellium.

Separating explicitely the two parts of the electric displacement, and adding the kinetic energy of the free elec- trons, one gets the following many-body Lagrangian:

$$
\begin{aligned}
L & =\sum_{i} \frac{m^{*}}{2} \dot{r}_{i}^{2}+\frac{2 \pi \tilde{\varepsilon}}{\omega_{L O}^{2}} \int\left(\dot{P}_{i r}^{2}-\omega_{L O}^{2} P_{i r}^{2}\right) \\
& -\frac{1}{8 \pi \varepsilon_{\infty}} \int \mathbf{D}^{+} \cdot \mathbf{D}^{+}-\frac{1}{8 \pi \varepsilon_{\infty}} \int \sum_{i \neq j} \mathbf{D}_{i}^{-} \cdot \mathbf{D}_{j}^{-} \\
& -\frac{1}{4 \pi \varepsilon_{\infty}} \int \mathbf{D}^{+} \cdot\left(\sum_{i} \mathbf{D}_{i}^{-}\right)+\int \mathbf{P}_{i r} \cdot\left(\sum_{i} \mathbf{D}_{i}^{-}+\mathbf{D}^{+}\right)
\end{aligned}
$$

The interacting terms in (5) now correspond to the selfenergy of the jellium, the Coulomb interactions among different electrons, the electron-jellium, electron-phonon and jellium-phonon interactions. Since the jellium is assumed to be rigid, it is convenient to introduce the polarization field which only responds to the electron motion by making use of the following transformation:

$$
\tilde{\mathbf{P}}_{i r}=\mathbf{P}_{i r}-\frac{1}{4 \pi \tilde{\varepsilon}} \mathbf{D}^{+}
$$

so that (5) becomes:

$$
\begin{aligned}
& L=\sum_{i} \frac{m^{*}}{2} \dot{r}_{i}^{2}+\frac{2 \pi \tilde{\varepsilon}}{\omega_{L O}^{2}} \int\left(\tilde{P}_{i r}^{2}-\omega_{L O}^{2} \tilde{P}_{i r}^{2}\right) \\
& -\frac{1}{8 \pi \varepsilon_{s}} \int \mathbf{D}^{+} \cdot \mathbf{D}^{+}-\frac{1}{8 \pi \varepsilon_{\infty}} \int \sum_{i \neq j} \mathbf{D}_{i}^{-} \cdot \mathbf{D}_{j}^{-} \\
& -\frac{1}{4 \pi \varepsilon_{s}} \int \mathbf{D}^{+} \cdot\left(\sum_{i} \mathbf{D}_{i}^{-}\right)+\int \tilde{\mathbf{P}}_{i r} \cdot\left(\sum_{i} \mathbf{D}_{i}^{-}\right)
\end{aligned}
$$

(the phonon-jellium interaction has disappeared, and the remaining terms involving the jellium are now divided by $\left.\varepsilon_{s}\right)$. The many-body Lagrangian in its final form (7) depends on the following parameters: (i) the electron density $n$; (ii) the parameters of the host material $m^{*}, \varepsilon_{s}, \varepsilon_{\infty}, \omega_{L O}$, which determine the e-ph coupling $\alpha$.

\subsection{Phonon integration}

The exact partition function of the system can be expressed in the path integral formulation as:

$$
Z=\operatorname{Tr}\left(e^{-\beta H}\right)=\int e^{S} \mathcal{D}(\text { path })
$$

where path integrals run over all the electron coordinates and on the polarization field. $S$ is defined as usual as the integral of $L$ in imaginary time $\left(t=i \tau, \beta^{-1}\right.$ being the temperature, and taking $\left.\hbar=k_{B}=1\right)$. The trace operation corresponds to integrating over all possible closed trajectories. Since the terms depending on the polarization are quadratic, the field $\tilde{\mathbf{P}}_{i r}$ can be exactly integrated out [18]. As a result, the total effective action for the electrons is the sum of three terms:

$$
S\left(\left\{\mathbf{r}_{i}\right\}\right)=S_{\text {jellium }}+S_{e}\left(\left\{\mathbf{r}_{i}\right\}\right)+S_{e-p h}\left(\left\{\mathbf{r}_{i}\right\}\right)
$$


The first term is constant at fixed density and does not depend on the state of the system:

$$
S_{\text {jellium }}=-\frac{\beta}{8 \pi \varepsilon_{s}} \int \mathbf{D}^{+} \cdot \mathbf{D}^{+}
$$

The second term in (9) is the electronic part:

$$
\begin{aligned}
S_{e}\left(\left\{\mathbf{r}_{i}\right\}\right) & =\sum_{i} \frac{m^{*}}{2} \int_{0}^{\beta} \dot{r}_{i}^{2} d \tau-\frac{1}{8 \pi \varepsilon_{\infty}} \int_{0}^{\beta} \int \sum_{i \neq j} \mathbf{D}_{i}^{-} \cdot \mathbf{D}_{j}^{-} d \tau \\
& -\frac{1}{4 \pi \varepsilon_{s}} \int_{0}^{\beta} \int \mathbf{D}^{+} \cdot\left(\sum_{i} \mathbf{D}_{i}^{-}\right) d \tau
\end{aligned}
$$

It contains the electron kinetic energy, the instantaneous Coulomb repulsion between the electrons, and the interaction between the electrons and the jellium.

The electron-phonon coupling effects are included in $S_{e-p h}$, which is given by:

$$
S_{e-p h}\left(\left\{\mathbf{r}_{i}\right\}\right)=\sum_{i, j} \frac{\omega_{L O} e^{2}}{4 \tilde{\varepsilon}} \int_{0}^{\beta} \int_{0}^{\beta} \frac{G_{\omega_{L O}}(\beta, \tau-\sigma)}{\left|\mathbf{r}_{i}(\tau)-\mathbf{r}_{j}(\sigma)\right|} d \tau d \sigma
$$

We have introduced the phonon propagator $G_{\omega}(\beta, \tau-\sigma)=$ $(\bar{n}+1) e^{-\omega|\tau-\sigma|}+\bar{n} e^{\omega|\tau-\sigma|}$, together with $\bar{n}=\left(e^{\beta \omega}-1\right)^{-1}$. Formula (12) represents the retarded interactions between electrons, mediated by the lattice polarization: the diagonal terms $(i=j)$ correspond to the interaction of each electron with itself, i.e. the polaron effect, while the offdiagonal terms $(i \neq j)$ give a retarded attraction between electrons $i$ and $j$.

The above expressions (9)-(12) are valid at any temperature. We will now apply our hypothesis of crystallization at low densities, and make use of several approximations.

\subsection{Model for the Crystallized State}

Let us first consider the jellium. We will assume from now on that it is constitued of spheres of radius $R_{s}$ with a uniform positive charge density $\rho^{+}=e /\left(4 \pi R_{s}^{3} / 3\right)$. Each sphere, which carries a total charge $+e$, is supposed to be centered on the sites $\left\{\mathbf{R}_{i}\right\}$ of a Bravais lattice. This approximation is intermediate between considering actual doping ion potentials, and a jellium uniformly spread out in the system. There is a small overlap between adjacent spheres, that we will neglect in the evaluation of the electrostatic energy. This is convenient to evaluate the jelliumjellium and the electron-jellium interaction terms. In particular, one can write:

$$
\mathbf{D}^{+}(\mathbf{r})=\sum_{i} \mathbf{D}_{i}^{+}(\mathbf{r})
$$

where

$$
\mathbf{D}_{i}^{+}(\mathbf{r})=\left\{\begin{array}{l}
\frac{e}{4 \pi R_{s}^{3}}\left(\mathbf{r}-\mathbf{R}_{i}\right) ; \text { if }\left|\mathbf{r}-\mathbf{R}_{i}\right|<R_{s} \\
\frac{e}{4 \pi} \frac{\mathbf{r}-\mathbf{R}_{i}}{\left|\mathbf{r}-\mathbf{R}_{i}\right|^{3}} \quad ; \text { otherwise }
\end{array}\right.
$$

so that

$$
\begin{aligned}
\frac{1}{8 \pi \varepsilon_{s}} \int \mathbf{D}_{i}^{+} \cdot \mathbf{D}_{i}^{+} & =\frac{3 e^{2}}{5 \varepsilon_{s} R_{s}} \\
\frac{1}{8 \pi \varepsilon_{s}} \sum_{i \neq j} \int \mathbf{D}_{i}^{+} \cdot \mathbf{D}_{j}^{+} & =\frac{1}{2} \sum_{i \neq j} \frac{e^{2}}{\varepsilon_{s}\left|\mathbf{R}_{i}-\mathbf{R}_{j}\right|}
\end{aligned}
$$

We next assume that the electrons are localized around the sites $\mathbf{R}_{i}$ of the same Bravais lattice, and we introduce the small displacements $\mathbf{u}_{i}=\mathbf{r}_{i}-\mathbf{R}_{i}$, so that

$$
\begin{aligned}
\frac{1}{4 \pi \varepsilon_{s}} \int_{(i \neq j)} \mathbf{D}_{i}^{+} \cdot \mathbf{D}_{j}^{-} & =-\frac{e^{2}}{\varepsilon_{s}\left|\mathbf{R}_{i}-\mathbf{R}_{j}-\mathbf{u}_{j}\right|} \\
\frac{1}{4 \pi \varepsilon_{s}} \int \mathbf{D}_{i}^{+} \cdot \mathbf{D}_{i}^{-} & =-\frac{3 e^{2}}{2 \varepsilon_{s} R_{s}}+\frac{m^{*}}{2} \frac{\omega_{W}^{2}}{\varepsilon_{s}} u_{i}^{2}
\end{aligned}
$$

where we have defined $\omega_{W}^{2}=e^{2} / m^{*} R_{s}^{3}=\omega_{p}^{2} / 3, \omega_{p}$ being the electron plasma frequency. Both $S_{e}$ and $S_{e-p h}$ can be decomposed into diagonal $(i=j)$ and off-diagonal $(i \neq j)$ terms, i.e.:

$$
\begin{aligned}
& S_{e}=-\frac{e^{2}}{2 \varepsilon_{\infty}} \sum_{i \neq j} \int_{0}^{\beta} \frac{1}{\left|\mathbf{R}_{i}-\mathbf{R}_{j}+\mathbf{u}_{i}(\tau)-\mathbf{u}_{i}(\tau)\right|} d \tau \\
& \quad-\sum_{i} \int_{0}^{\beta}\left[\frac{9 e^{2}}{10 \varepsilon_{s} R_{s}}+\frac{m^{*}}{2} \dot{u}_{i}^{2}(\tau)+\frac{m^{*}}{2} \frac{\omega_{W}^{2}}{\varepsilon_{s}} u_{i}^{2}(\tau)\right] d \tau
\end{aligned}
$$

and

$$
\begin{aligned}
& S_{e-p h}=\sum_{i} \frac{\omega_{L O} e^{2}}{4 \tilde{\varepsilon}} \int_{0}^{\beta} \int_{0}^{\beta} \frac{G_{\omega_{L O}}(\beta, \tau-\sigma)}{\left|\mathbf{u}_{i}(\tau)-\mathbf{u}_{i}(\sigma)\right|} d \tau d \sigma \\
& +\sum_{i \neq j} \frac{\omega_{L O} e^{2}}{4 \tilde{\varepsilon}} \int_{0}^{\beta} \int_{0}^{\beta} \frac{G_{\omega_{L O}}(\beta, \tau-\sigma)}{\left|\mathbf{R}_{i}-\mathbf{R}_{j}+\mathbf{u}_{i}(\tau)-\mathbf{u}_{i}(\sigma)\right|} d \tau d \sigma
\end{aligned}
$$

Our next approximation consists in expanding the action up to second order in the $\left\{\mathbf{u}_{i}\right\}$, thus ignoring all the anharmonic and higher order contributions. After some elementary algebra, one gets:

$$
S\left(\left\{\mathbf{u}_{i}\right\}\right)=\sum_{i} S_{i}+\frac{1}{2} \sum_{i \neq j} S_{i j}
$$

with

$$
\begin{aligned}
S_{i}= & -\beta \frac{9 e^{2}}{10 \varepsilon_{s} R_{s}}-\int_{0}^{\beta}\left[\frac{m^{*}}{2} \dot{u}_{i}^{2}(\tau)+\frac{m^{*}}{2} \frac{\omega_{W}^{2}}{\varepsilon_{s}} u_{i}^{2}(\tau)\right] d \tau \\
& +\frac{\omega_{L O} e^{2}}{4 \tilde{\varepsilon}} \int_{0}^{\beta} \int_{0}^{\beta} \frac{G_{\omega_{L O}}(\beta, \tau-\sigma)}{\left|\mathbf{u}_{i}(\tau)-\mathbf{u}_{i}(\sigma)\right|} d \tau d \sigma
\end{aligned}
$$

and

$$
\begin{aligned}
& S_{i j}=-\frac{e^{2}}{\varepsilon_{\infty}} \sum_{\alpha \gamma} \int_{0}^{\beta} \Lambda_{i j}^{\alpha \gamma} u_{i}^{\alpha}(\tau) u_{j}^{\gamma}(\tau) d \tau \\
& +\frac{\omega_{L O} e^{2}}{2 \tilde{\varepsilon}} \sum_{\alpha \gamma} \int_{0}^{\beta} \int_{0}^{\beta} \Lambda_{i j}^{\alpha \gamma} G_{\omega_{L O}}(\beta, \tau-\sigma) u_{i}^{\alpha}(\tau) u_{j}^{\gamma}(\sigma) d \tau d \sigma
\end{aligned}
$$


where the indices $\alpha, \gamma=(x, y, z)$ denote the cartesian coordinates. We have also defined the dipolar matrix elements:

$$
\Lambda_{i j}^{\alpha \gamma}=\frac{\delta_{\alpha \gamma} R_{i j}^{2}-3 R_{i j}^{\alpha} R_{i j}^{\gamma}}{R_{i j}^{5}}
$$

Expressions (16)-(18) constitute our basic model for the polaron crystal. Since we focus on the insulating state at low density, the action $S$ can be treated semi-classically, i.e. neglecting the exchange between the fermions and quantum statistical effects.

\subsection{Comparison with the electron crystal}

Our model (16) was obtained by making use of the following approximations: (i) we consider a particular structure for the jellium, (ii) we expand the off-diagonal part of the action up to second order in the displacements $\mathbf{u}_{i}$, and (iii) we neglect the exchange between different electrons. In order to analyse in more detail the consequences of these assumptions, we can compare our results with the previous treatments of the electron crystal. Carr [20] demonstrated that the energy per electron in a WC with bcc symetry can be expressed as an expansion in powers of $R_{s}^{-1 / 2}$. Introducing the dimensionless density parameter $r_{s}=R_{s} / a_{0}$ ( $a_{0}=\hbar^{2} / m_{e} e^{2} \approx 0.53 \AA$ is the Bohr radius) and taking the Rydberg as the energy unit, the result reads

$$
E=\frac{-1.792}{r_{s}}+\frac{2.66}{r_{s}^{3 / 2}}+\frac{b}{r_{s}^{2}}+O\left(r_{s}^{5 / 2}\right)+O\left(e^{-r_{s}^{1 / 2}}\right)
$$

with $b<1$. Basically, our three approximations above consist in neglecting all the terms in powers of $r_{s}$ lower than $-3 / 2$ in (20). In fact, the first assumption slightly overestimates the Madelung energy, whose numerical value is lowered from $-1.792 / r_{s}$ to $-1.8 / r_{s}$. The second hypothesis consists in neglecting the anharmonic $\left(b / r_{s}^{2}\right)$ and higher order terms. These terms can become important close to the melting point, and can change its numerical determination, but do not affect qualitatively the physics of the problem. Our last approximation is to neglect all the terms proportional to the overlap between the wavefunctions of different localized electrons. The corresponding energy terms in 20), i.e. the exchange terms, fall off exponentially with $r_{s}^{1 / 2}$. As pointed out by Carr, they are negligible up to $r_{s} \approx 10$. In the polaron crystal, where localization is more efficient due to the e-ph coupling, it is quite reasonable to neglect them in the density range of interest (these terms are responsible for the magnetic properties of the crystal, that we do not consider here).

\section{The mean-field Wigner approximation: solution by the Feynman method}

In the following, we will restrain ourselves to the diagonal part of the action $S=\sum_{i} S_{i}$, neglecting the dipolar terms $S_{i j}$. This is generally called the Wigner approximation. It is sensitive since, using Gauss' theorem, it can be easily shown that the total mean electric field of a particular sphere (averaged in time), including the jellium, the electron, and the polarization field, vanishes for $\left|\mathbf{r}_{i}-\mathbf{R}_{i}\right|>R_{s}$ [6]. In other words, two different Wigner spheres can only interact through the deviations of $\mathbf{r}_{i}$ from equilibrium, and that goes beyond the mean-field approximation. Each sphere is then on the average an independent entity, and the effect of the other particles is already included in the harmonic potential of eq. (15). At this stage, all the electrons localized in their jellium spheres are equivalent and uncoupled, so that the system is fully described by any of the single-particle actions (17) - i.e. we can take $S=S_{i}$. As was stated in the introduction, the off-diagonal elements $S_{i j}$ will be treated in a further publication.

The retarded interaction in (17), which is responsible for polaron formation, cannot be integrated exactly. Following Feynman 18], we introduce a quadratic trial action of the form

$$
\begin{aligned}
S_{0} & =-\frac{m^{*}}{2} \int \dot{u}^{2} d \tau-\frac{m^{*}}{2} \frac{\omega_{W}^{2}}{\varepsilon_{s}} \int u^{2} d \tau \\
& -\frac{K w}{8} \iint|\mathbf{u}(t)-\mathbf{u}(s)|^{2} G_{w}(\beta, \tau-\sigma) d \tau d \sigma
\end{aligned}
$$

whose parameters $K$ and $w$ will be determined variationally (here $\mathbf{u}_{i}$ has been replaced by the coordinate $\mathbf{u}$ of the only electron present). It can be seen that (21) corresponds to the following two-body Lagrangian

$$
L_{0}=\frac{m^{*}}{2} \dot{u}^{2}+\frac{M}{2} \dot{X}^{2}-\frac{K}{2}(\mathbf{u}-\mathbf{X})^{2}-\frac{m^{*}}{2} \frac{\omega_{W}^{2}}{\varepsilon_{s}} u^{2} .
$$

after the integration of $\mathbf{X}$ has been carried out. This model describes an electron subject to an external harmonic potential and bound with a spring to a fictitious particle of mass $M$ and coordinate $\mathbf{X}$. The latter represents the polarization cloud associated to the electron (see Fig.11). The constant $K$, which measures the strength of the retarded attraction, can be written as $K=M w^{2}$.

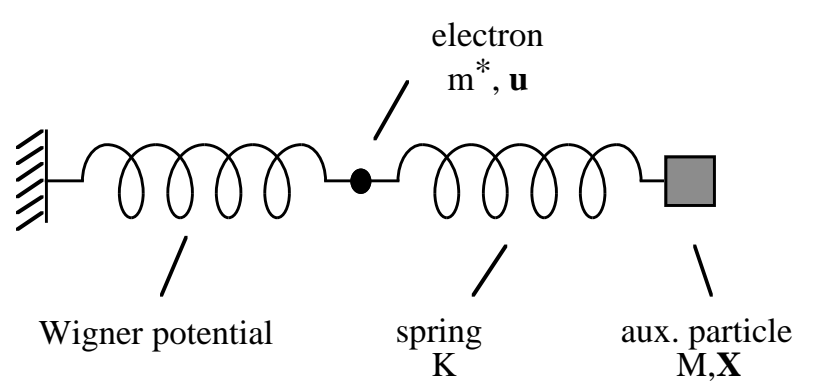

Fig. 1. The classical model corresponding to the action (21): the polarization cloud is replaced by a fictitious particle of mass $M$.

The Lagrangian (22) can be diagonalized into two harmonic modes $\mathbf{u}_{1}$ and $\mathbf{u}_{2}$, whose eigenfrequencies $\alpha_{1}$ and 
$\alpha_{2}$ are given by:

$$
\alpha_{1,2}^{2}=\frac{v^{2}+\omega_{W}^{2} / \varepsilon_{s}}{2} \mp \frac{\sqrt{\left(v^{2}+\omega_{W}^{2} / \varepsilon_{s}\right)^{2}-4 w^{2} \omega_{W}^{2} / \varepsilon_{s}}}{2}
$$

We have introduced the shortcut notation $v=(K / \mu)^{1 / 2}$ for the characteristic frequency of the two-body model ( $\mu=m^{*} M /\left(m^{*}+M\right)$ is the reduced mass). The two eigenfrequencies obey the following relations:

$$
\begin{aligned}
\alpha_{1}^{2}+\alpha_{2}^{2} & =v^{2}+\omega_{W}^{2} / \varepsilon_{s} \\
\alpha_{1} \alpha_{2} & =w \omega_{W} / \sqrt{\varepsilon_{s}} \\
\alpha_{1} & \leq w \leq \alpha_{2} .
\end{aligned}
$$

Finally, the mass $M$ of the auxiliary particle can be written as:

$$
\frac{M}{m^{*}}=\left(1-\frac{\alpha_{1}^{2}}{w^{2}}\right)\left(\frac{\alpha_{2}^{2}}{w^{2}}-1\right)
$$

and the polaron mass is defined as:

$$
M_{P}=m^{*}+M .
$$

If we introduce the quantities

$$
A_{1}=\frac{w^{2}-\alpha_{1}^{2}}{\alpha_{2}^{2}-\alpha_{1}^{2}}, A_{2}=\frac{\alpha_{2}^{2}-w^{2}}{\alpha_{2}^{2}-\alpha_{1}^{2}},
$$

the relation between the real coordinates and the normal modes is simply:

$$
\begin{aligned}
\mathbf{u} & =A_{1} \mathbf{u}_{1}+A_{2} \mathbf{u}_{2} \\
\mathbf{X} & =\frac{w^{2}}{\alpha_{2}^{2}-\alpha_{1}^{2}}\left(\mathbf{u}_{1}-\mathbf{u}_{2}\right) .
\end{aligned}
$$

and the diagonalized Lagrangian can be expressed in canonical form:

$$
L_{0}=\frac{1}{2} A_{1} \dot{u}_{1}^{2}+\frac{1}{2} A_{2} \dot{u}_{2}^{2}-\frac{1}{2} A_{1} \alpha_{1}^{2} u_{1}^{2}-\frac{1}{2} A_{2} \alpha_{2}^{2} u_{2}^{2} .
$$

\subsection{The low density regime}

At very low densities $\left(r_{s} \rightarrow \infty, \omega_{W} \approx 0\right)$, the polarons are so far apart that their properties are unchanged from the single polaron case. In other words, the localizing potential $V(\mathbf{u})=m^{*} \omega_{W}^{2} u^{2} / 2 \varepsilon_{s}$ acting on the electron is a perturbation with respect to the polaron energy, and the eigenfrequencies (23) can be expanded for small $\omega_{W}^{2} / \varepsilon_{s}$, which gives:

$$
\begin{aligned}
& \alpha_{1} \rightarrow \sqrt{\frac{m^{*}}{M_{P}}} \cdot \frac{\omega_{W}}{\sqrt{\varepsilon_{s}}} \equiv \omega_{e x t} \\
& \alpha_{2} \rightarrow v .
\end{aligned}
$$

( $\omega_{\text {ext }}$ is defined as the frequency of vibration of a particle of mass $M_{P}$ in a harmonic potential whose spring constant is $m^{*} \omega_{W}^{2} / \varepsilon_{s}$, while $v$ is the internal frequency of an isolated polaron [21]). The corresponding eigenmodes are:

$$
\begin{aligned}
& \mathbf{u}_{1} \rightarrow \mathbf{R}=\frac{m^{*} \mathbf{u}+M \mathbf{X}}{M_{P}} \\
& \mathbf{u}_{2} \rightarrow \mathbf{r}=\mathbf{u}-\mathbf{X} .
\end{aligned}
$$

These two independent modes correspond respectively to the vibration of the polaron center-of-mass, and to the vibration of the electron inside the polarization potential well. We shall call them respectively the external and the internal degree of freedom. It can be seen from expressions (29) that in the dilute regime, the external and internal energy scales are well separated, and the polaron can follow the vibrations imposed by the external potential as if it was a rigid particle. Basically, $\alpha_{1}$ and $\alpha_{2}$ give the excitation spectrum of the polaron crystal in the framework of the Wigner approximation, as is sketched in Fig.2. (note that the electron can also be excited towards a free

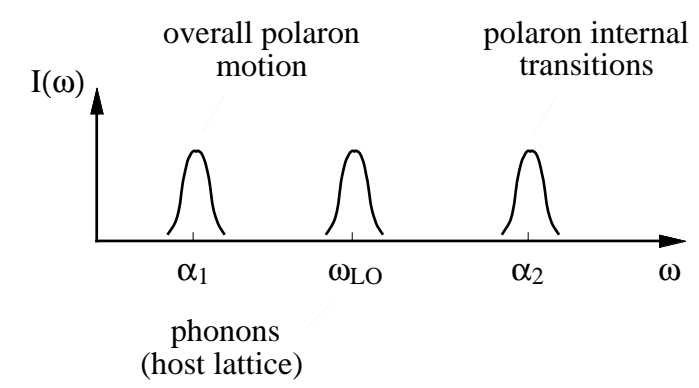

Fig. 2. Schematic absorption spectrum of the polaron crystal within Wigner's approximation. The two energy scales $\alpha_{1}$ and $\alpha_{2}$ are well separated, and the (host) phonon frequency lies in between.

state outside the polaron potential; the corresponding frequency, which is higher than $\alpha_{2}$, is not shown here).

\subsection{The crossover regime}

As was noted in the previous section, the localizing Wigner potential forces the polaron center-of-mass to vibrate with a frequency $\omega_{\text {ext }}$, which increases with increasing density. However, the polaron is a composite particle, and its velocity is physically limited by the characteristic frequency of the phonon cloud: when $\omega_{\text {ext }}$ approaches $\omega_{L O}$, the phonons are too slow to "follow" the overall motion, and only the electronic degree of freedom oscillates. In that case, both $\alpha_{1}$ and $\alpha_{2}$ can deviate significantly from their asymptotic values (29). If the density is further increased, the excess kinetic energy is transferred to the internal degree of freedom and the concept of a rigid polaron vibrating in an external potential breaks down. The crossover density is roughly given by the condition:

$$
\omega_{e x t} \approx \omega_{L O}
$$

that we express using (29) as:

$$
\left(\frac{m^{*}}{m_{e}}\right) \frac{r_{s}}{\varepsilon_{\infty}} \approx\left[\left(\frac{m^{*}}{M_{P}}\right) \frac{4 \eta}{(1-\eta)^{4}} \alpha^{4}\right]^{1 / 3},
$$


where $\eta=\varepsilon_{\infty} / \varepsilon_{s}$. In the limit of strong e-ph coupling $(\alpha \gtrsim 6)$, the mass of an isolated polaron is well approximated by $M_{P} / m^{*} \approx 0.02 \alpha^{4}$ [18, 26], so that eq. (31) can be expressed as:

$$
\left(\frac{m^{*}}{m_{e}}\right) \frac{r_{s}}{\varepsilon_{\infty}} \approx\left[200 \frac{\eta}{(1-\eta)^{4}}\right]^{1 / 3}
$$

and the crossover density becomes independent on the eph coupling. As an example, if we take $m^{*}=m_{e}, \varepsilon_{s}=30$ and $\varepsilon_{\infty}=5$, the crossover region corresponds to $r_{s} \approx 20$. In the opposite limit $(\alpha \rightarrow 0)$, the phonon frequency tends to infinity, so that $\omega_{\text {ext }}$ never reaches the value $\omega_{L O}$. In that case, the internal structure of the polarons can safely be neglected, and the polaron crystal tends to an ordinary electron crystal.

\subsection{Results of the variational procedure}

As in the case of the single polaron problem, Feynman's variational method gives, for any $\alpha$, the best analytical upper bound for the free energy $F$. This is obtained by minimizing the following expression:

$$
F=F_{0}-\frac{1}{\beta}\left\langle S-S_{0}\right\rangle
$$

where the term $F_{0}$ is defined as

$$
e^{-\beta F_{0}}=\int \mathcal{D}(\text { path }) e^{S_{0}} .
$$

and $\langle\cdots\rangle$ stands for $\int \mathcal{D}($ path $)(\ldots) e^{S_{0}} / \int \mathcal{D}($ path $) e^{S_{0}}($ path integrations must be carried out on closed trajectories). The calculation of the functional $(33)$ to be minimized follows the lines of Feynman's work (details are presented in the Appendix). The ground-state energy $E$ at zero temperature can be obtained by taking the limit $\beta \rightarrow \infty$ in the preceding expressions. The result is:

$$
\begin{gathered}
E=C_{0}+\frac{3}{2}\left(\alpha_{1}+\alpha_{2}-w\right)-\frac{3\left(w^{2}-\alpha_{1}^{2}\right)\left(\alpha_{2}^{2}-w^{2}\right)}{4\left(\alpha_{1}+\alpha_{2}\right) w^{2}} \\
-\frac{\alpha}{\sqrt{\pi}} \int_{0}^{\infty} \frac{e^{-t} d t}{\sqrt{\frac{A_{1}}{\alpha_{1}}\left(1-e^{-\alpha_{1} t}\right)+\frac{A_{2}}{\alpha_{2}}\left(1-e^{-\alpha_{2} t}\right)}}
\end{gathered}
$$

where all frequencies are expressed in units of $\omega_{L O}$, and $C_{0}=-\left(9 \tilde{\varepsilon}^{2} \alpha^{2} / 5 \varepsilon_{s} r_{s}\right)\left(m_{e} / m^{*}\right)$. The last integral is non elementary and must be calculated numerically. $\alpha_{1,2}$ are taken as the two independent variational parameters, and $w$ is related to $\alpha_{1,2}$ through (24). The expression (35), after minimization, represents the energy per particle of the PWC in the Wigner approximation. The result is sketched in Fig. 3 for different values of $\alpha$. It is interesting to note that, upon increasing $\alpha$, the evolution from a WC of electrons towards a PWC is gradual. In fact, all the curves in Fig 3 have the same behaviour, and the only difference comes from the fact that the minimum of $E$ becomes more pronounced and shifts to higher densities. This is not surprising, since it is known that the formation of large polarons is not a real phase transition, but rather a continuous crossover [22].

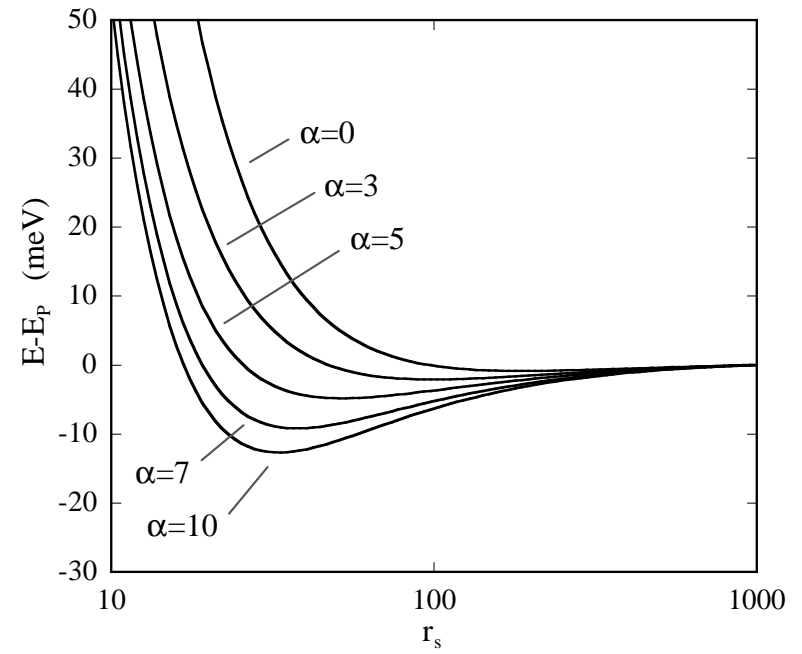

Fig. 3. The energy per polaron in the crystallized state versus $r_{s}$ (the single polaron value $E_{P}$, corresponding to the limit $r_{s}=\infty$, has been subtracted out). From top to bottom $\alpha=0$ (electron crystal), $\alpha=3,5,7,10$. The energy unit is $m e V$. In this and the following figures, the parameters of the host lattice are $\varepsilon_{s}=30, \varepsilon_{\infty}=5, m^{*}=m_{e}$.

\subsubsection{Eigenfrequencies}

The three frequencies $w, \alpha_{1}$ and $\alpha_{2}$ are illustrated in Fig.(1) as a function of the density, for two different values of $\alpha$. As noted above, at weak e-ph coupling, the phonon cloud is fast and it can easily follow the polaron motion at any density. The external frequency $\alpha_{1}$ is proportional to $\omega_{W}$, while $\alpha_{2}$, which tends to $w$ in this limit, is almost density independent (see inset). The two frequencies are always well separated, and the polaron can vibrate as if it was a rigid particle.

On the other hand, at intermediate and strong $\alpha$, the internal and external degrees of freedom are fully decoupled only at low density (see main plot). When we reach the crossover region (indicated by an arrow), the external frequency "saturates" around $\omega_{L O}$ : the phonon cloud cannot follow the vibrations imposed by the Wigner potential, and the polaron center-of-mass is virtually frozen. Upon increasing the density, the excess kinetic energy is thus transferred to the internal degree of freedom, and $\alpha_{2}$ becomes proportional to $\omega_{W}$.

\subsubsection{Polaron mass}

In Fig. f we illustrate the behaviour of $M_{P}$ as a function of $r_{s}$, for different values of $\alpha$. It can be seen that $M_{P}$ always increases with the density when one approaches the crossover regime. A similar effect is well-known for an isolated polaron moving at a finite velocity $\mathrm{v}$ [23]. If the quasi-particle kinetic energy $M_{P} \mathrm{v}^{2} / 2$ reaches the phonon energy, the effective polaron mass increases because phonons can be emitted from the polarization cloud in an incoherent way (the equivalent of Cherenkov effect in transparent media). If we consider a polaron in a Wigner 


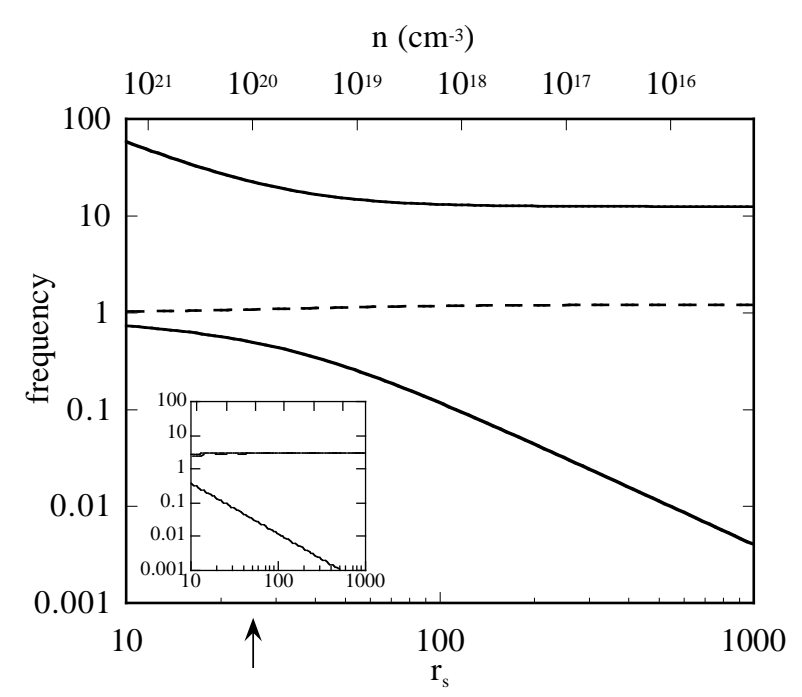

Fig. 4. The characteristic frequencies of the two-body model (22), in units of $\omega_{L O}: \alpha_{2}$ (upper curve), $w$ (dashed curve), $\alpha_{1}$ (bottom curve). The e-ph coupling is $\alpha=10$. The arrow marks the crossover region (see sec. 3.2). Inset: same as main plot, with $\alpha=1$.

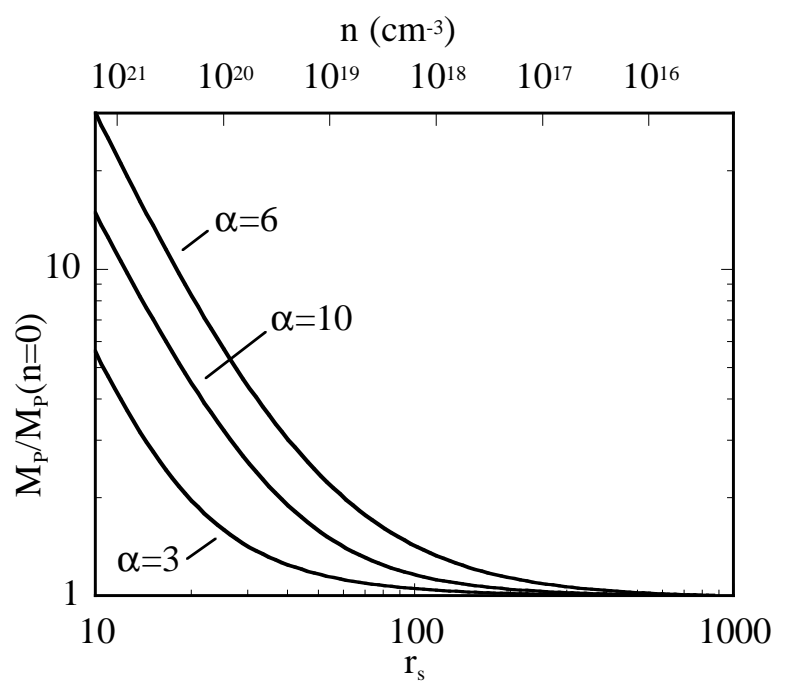

Fig. 5. The polaron mass $M_{P}$, normalized by the single polaron value, for $\alpha=3,6,10$.

sphere, whose average velocity is $\mathrm{v} \approx \omega_{\text {ext }} \sqrt{\hbar / M_{P} \omega_{\text {ext }}}$, it is easy to verify that the condition $M_{P} \mathrm{v}^{2} / 2 \approx \hbar \omega_{L O}$ gives exactly the crossover condition (31).

\section{The insulator-to-metal transition}

The melting of the ordinary electron crystal at zero temperature has been extensively studied in the literature, and there exist many different criteria which allow to evaluate the critical density for the transition (see Care 24 for a review). A particularly simple approach was applied by Nozières and Pines [19], which is based on the Lindemann criterion. This phenomenological criterion, generally used to describe the thermal melting of the atomic lattices, states that the crystal is unstable when the spatial fluctuations of each particle around its equilibrium position exceed some fraction of the inter-particle distance. Such criterion will be generalized here to the case of a PWC, and will be used at both zero and finite temperature in order to describe qualitatively the insulator-to-metal transition.

\subsection{Lindemann Criterion}

Calling $\mathbf{u}=(x, y, z)$ the coordinate of a localized electron in an ordinary Wigner crystal, the Lindemann criterion states that the crystal melts when

$$
\left\langle\delta u^{2}\right\rangle^{1 / 2} / R_{s}>\delta
$$

where $\delta$ is a phenomenological constant usually taken as $\delta \approx 0.25$. In the Wigner approximation, the average zeropoint displacement in each space direction ( say $x$ ) is given at zero temperature by:

$$
\left\langle\delta x^{2}\right\rangle^{1 / 2}=\left(\frac{\hbar}{2 m_{e} \omega_{W}}\right)^{1 / 2} .
$$

If we consider three dispersionless modes of equal frequency $\omega_{W}=\omega_{p} / \sqrt{3}$, as is the case if we neglect dipoledipole interactions, we get a critical density parameter $r_{c}^{W}=64$, which is close to the Monte-Carlo result $\left(r_{c}^{W}=\right.$ $100 \pm 20$ in $3 \mathrm{D}, 25]$ ). Of course, this criterion is only qualitative and the resulting melting density is very sensitive to the choice of $\delta$, and moreover it does not give any precise information about the nature of the ground-state beyond the melting point. However, especially in the polaronic case, we shall see that it gives a good insight in the physics of the problem.

The treatment that we have described in the previous sections, which reduces the many-polaron problem to the problem of a single polaron in an effective potential, gives reliable results for the insulating state at any value of the e-ph coupling. It was shown in section 3 that the system passes continuously from an ordinary Wigner lattice at weak $\alpha$ to a lattice of polarons at strong $\alpha$, with no symmetry breaking between the two limits. However, we will show here that a definite distinction between the two cases comes from the melting mechanisms. Indeed, taking into account the composite nature of the polarons, we can introduce two different Lindemann criteria, whether we analyse the fluctuation of the polaron as a whole, or rather the fluctuation of the electron with respect to the polaritazion field. Using the definitions (30) of the centerof-mass $\mathbf{R}$ and of the relative coordinate $\mathbf{r}$, we can write the following conditions:

$$
\begin{aligned}
& \text { (i) }\left\langle\delta R^{2}\right\rangle^{1 / 2} / R_{s}>0.25 \\
& \text { (ii) }\left\langle\delta r^{2}\right\rangle^{1 / 2} / R_{s}>0.25
\end{aligned}
$$

The former gives the critical value $r_{c}^{(i)}$ for the melting towards a polaron liquid. The latter gives the critical value 
$r_{c}^{(i i)}$ for polaron dissociation. The calculation of $\left\langle\delta R^{2}\right\rangle$ and $\left\langle\delta r^{2}\right\rangle$ in terms of the variational parameters is presented in the appendix. The result at $T=0$ is (all frequencies are expressed in units of $\left.\omega_{L O}\right)$ :

$$
\begin{aligned}
& \left\langle\delta R^{2}\right\rangle^{1 / 2} / R_{s}=\left(\frac{\omega_{W}^{2}}{4 \alpha}\right)^{1 / 3} \frac{m^{*}}{M_{P}} \sqrt{\frac{A_{1}}{\alpha_{1}}\left(\frac{\alpha_{2}}{w}\right)^{4}+\frac{A_{2}}{\alpha_{2}}\left(\frac{\alpha_{1}}{w}\right)^{4}} \\
& \left\langle\delta r^{2}\right\rangle^{1 / 2} / R_{s}=\left(\frac{\omega_{W}^{2}}{4 \alpha}\right)^{1 / 3} \frac{1}{\alpha_{2}^{2}-\alpha_{1}^{2}} \sqrt{\frac{\alpha_{1}^{2}}{A_{1}}+\frac{\alpha_{2}^{2}}{A_{2}}}
\end{aligned}
$$

When the e-ph coupling vanishes $\left(M_{P} \rightarrow m^{*}\right.$ and $\mathbf{R} \rightarrow$ $\mathbf{u}$ ), eq. ( $i$ ) reduces to the ordinary Lindemann criterion for the WC, and the transition is due to fluctuations of the (weakly renormalized) electrons. On the other hand, the fluctuation of the internal coordinate gives a measure of the polaron radius [26], so that criterion $(i i)$ can be written as $R_{P} / R_{s}=$ const, which closely resembles eq. (11) above. As a matter of fact, we can consider the second criterion as a generalization to finite phonon frequencies of the dissociation argument given in the introduction (which we have shown to be valid in the limit $\omega_{L O} \rightarrow 0$, when the potential acting on the electron is completely static).

\subsection{Transition at $\mathrm{T}=0$}

We are now in a position to analyse the IMT for any values of $\alpha$. Fig. 6 shows the calculated ratios which enter in
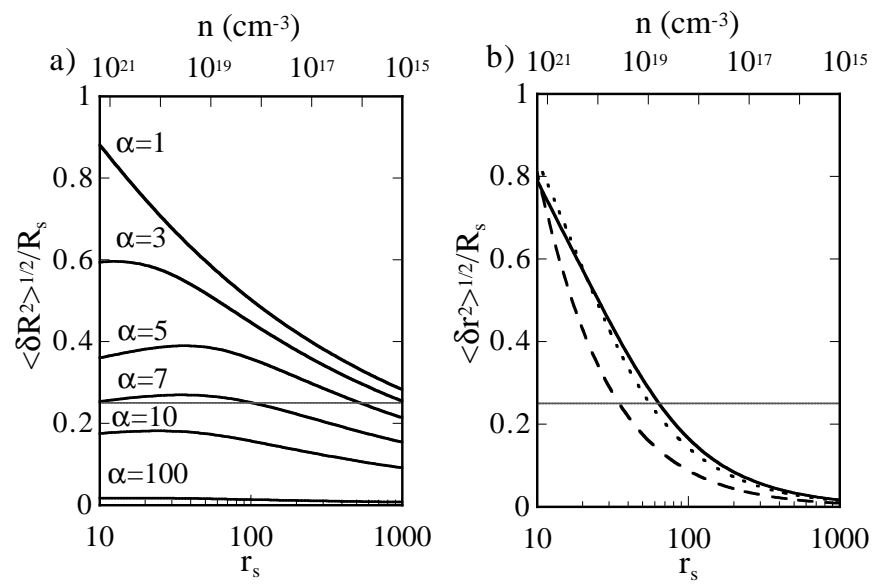

Fig. 6. The ratios which enter in the Lindemann criteria (i) and (ii): a) $\left\langle\delta R^{2}\right\rangle^{1 / 2} / R_{s}$ for $\alpha=1,3,5,7,10,100$; b) $\left\langle\delta r^{2}\right\rangle^{1 / 2} / R_{s}$ for $\alpha=1$ (dashed line), $\alpha=3$ (dotted line), $\alpha=10$ (full line). The horizontal line corresponds to the phenomenological melting value $\delta=0.25$.

the conditions $(i)$ and $(i i)$, as functions of $r_{s}$, for different values of $\alpha$. One observes that for a fixed $\alpha$, while $\left\langle\delta r^{2}\right\rangle^{1 / 2} / R_{s}$ always increases with increasing density (all the curves in Fig.6.b have roughly the same behaviour), the center-of-mass fluctuation $\left\langle\delta R^{2}\right\rangle^{1 / 2} / R_{s}$ becomes bell shaped for sufficiently large $\alpha$ (it changes its slope exactly where, according to the argument of section 3.2, the internal structure of the polaron becomes important - see Fig.6.a). In addition, the maximum value of $\left\langle\delta R^{2}\right\rangle^{1 / 2} / R_{s}$ decreases with increasing $\alpha$. Remembering that the transition occurs when one of the fluctuation ratios reaches the value 0.25 , we see that there exists a certain critical value $\alpha^{*}$ above which the crystal melting $\grave{a}$ la Wigner is prevented, and the driving mechanism switches to the polaron dissociation, typical of the static limit. An important consequence of this result is that, for $\alpha>\alpha^{*}$, the polarons do not survive beyond the IMT. In other words, in the strong e-ph coupling limit, a liquid state of large polarons cannot exist at zero temperature. With the parameters of Fig. 6, we find $\alpha^{*} \simeq 7.5$.

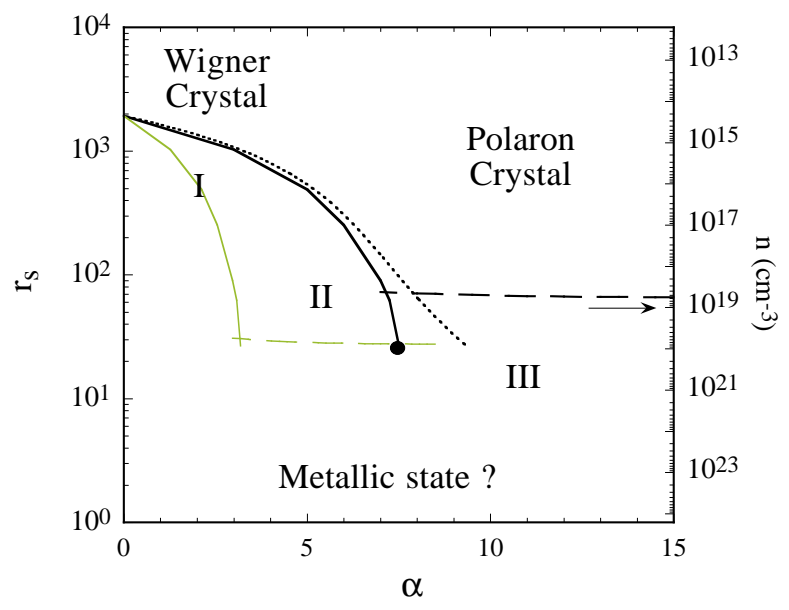

Fig. 7. Zero temperature phase diagram as deduced from criteria $(i)$ and $(i i): r_{c}^{(i)}$ for crystal melting (full line) and $r_{c}^{(i i)}$ for polaron dissociation in 3D (dashed line). The corresponding quantities for an anisotropic polaron crystal (see section 4.4) are drawn in grey. The dotted line corresponds to the low density approximation (38). The melting driven by the fluctuations of the localized particles (criterion $(i)$ ) is impossible beyond $\alpha^{*}$ (full circle). The critical density given by eq. (11) is indicated by an arrow. For regions I, II and III, see text.

Fig.77 illustrates the approximate phase diagram obtained from the two Lindemann criteria at $T=0$. The full line corresponds to criterion $(i)$. We see that $r_{c}^{(i)}$ decreases with increasing $\alpha$ (the corresponding critical density increases). This can be easily understood in terms of the asymptotic behaviour of section 3.1. As we have stated above, at low density, we can neglect the composite nature of the polaron and its mean square displacement in an external potential of frequency $\omega_{\text {ext }}$ is given by:

$$
\left\langle\delta R^{2}\right\rangle^{1 / 2} \simeq \sqrt{\frac{\hbar}{2 M_{P} \omega_{e x t}}},
$$

which fits very well the low density part of $\left\langle\delta R^{2}\right\rangle^{1 / 2} / R_{s}$ (right side of Fig. 6.(a)). In the weak coupling regime (in practice for all $\alpha<\alpha^{*}$ ), we can use this formula to calculate the critical $r_{s}$ for crystal melting. The result can 
be expressed in terms of the critical parameter $r_{c}^{W}$ of the ordinary electron crystal as:

$$
r_{c}^{(i)} \simeq r_{c}^{W} \varepsilon_{s} \frac{m_{e}}{M_{P}}
$$

The competition between the kinetic and potential energy is clearly visible in eq. (38): if the mass of the carriers is strong enough, despite the static screening of the charges which reduces the Coulomb interactions, the crystallization is favored with respect to the ordinary WC of electrons. The melting curve corresponding to this approximation is shown in Fig.7 (dotted line: we see that (38) slightly underestimates the critical density, especially for $\alpha$ close to $\left.\alpha^{*}\right)$. Since the polarons do not dissociate at the transition, we expect the metallic state beyond $n_{c}^{(i)}$ to be either a renormalized electron liquid (region I) or a degenerate polaron liquid (region II).

For $\alpha>\alpha^{*}$, the IMT is described by the dissociation mechanism $(i i)$, and the critical density is almost constant (in a nutshell, the crystal is destroyed when the polarons start to overlap). In this range, although the polarons do not survive beyond the transition, the resulting electron liquid should present both strong e-ph and e-e interactions (region III). A second possibility is that not all of the polarons are destroyed at the transition, because this would cost too much potential energy, especially in the strong coupling limit. This can be understood as follows: when the density of localized polarons is increased, there is a competition between the increase of $\left\langle\delta r^{2}\right\rangle^{1 / 2} / R_{s}$ due to the zero-point fluctuations (which destabilizes the PWC), and the tendency of the system to preserve the potential energy stored in the polaron bound states (which in turn should prevent dissociation). A compromise could be achieved in a hypothetical mixed phase, where some itinerant carriers could coexist with the localized polarons. In fact, some experiments [27] suggest that all the carriers introduced beyond the critical density are itinerant (their number being proportional to $n-n_{c}^{(i i)}$ ), the density of localized polarons being kept constant and equal to $n_{c}^{(i i)}$. The properties of such a mixed phase are currently under studies. It is clear, however, that this could be stable only close to $n_{c}^{(i i)}$, while for $n \gg n_{c}^{(i i)}$, all the interactions would be screened, and the system would tend to a normal Fermi liquid.

\subsection{Transition at $T \neq 0$}

If we go to finite temperatures, the melting of the polaron crystal can be studied in terms of the same Lindemann criteria $(i)$ and $(i i)$ as in the zero temperature case, provided that we include the effect of thermal fluctuations in the definition of $\left\langle\delta R^{2}\right\rangle$ and $\left\langle\delta r^{2}\right\rangle$. The correct result is given in the appendix (see eq. (70) and (71)), where the variational parameters $\alpha_{1}$ and $\alpha_{2}$ are now obtained by minimizing the free energy $F$ given by eq. (48), together with (51), (52) and (66). It is known from the single polaron case, however, that the upper bound (33) that one estimates starting from the two-body model (22) only gives correct results for $T<\omega_{L O}$ 228,16]. At higher temperatures, such quantities as the polaron mass $M_{P}$ and internal frequency $\alpha_{2}$ are ill-defined, and the treatment is no longer applicable in this simple form. Nevertheless, since the characteristic energy scale for the thermal fluctuations of the polaron center-of-mass is $\alpha_{1}<\omega_{L O}$, we reasonably expect the thermal melting of the crystal to occur at temperatures well beyond the limits of validity of the treatment, as can be verified a posteriori.

In order to understand the basic physics of the problem, we first restrict ourselves to the low-density regime as was done in section 3.1. This allows us to express $\left\langle\delta R^{2}\right\rangle$ and $\left\langle\delta r^{2}\right\rangle$ by the approximate formulas

$$
\begin{aligned}
\left\langle\delta R^{2}\right\rangle & =\left(2 M_{P} \alpha_{1}\right)^{-1} \operatorname{coth} \frac{\beta \alpha_{1}}{2} \\
\left\langle\delta r^{2}\right\rangle & =\left(2 \mu \alpha_{2}\right)^{-1} \operatorname{coth} \frac{\beta \alpha_{2}}{2} .
\end{aligned}
$$

Polaron theory predicts that at temperatures much lower than $\omega_{L O}$, the properties of each polaron are almost unaffected by thermal fluctuations 28, 26]. In that case, we can consider to a first approximation $\alpha_{2}(T) \approx \alpha_{2}, M_{P}(T) \approx$ $M_{P}$, and consequently $\alpha_{1}(T) \approx \alpha_{1}$ (from eq. (24)). Therefore, in this simple limit, the only temperature dependence of the radii (39) and (40) comes from the explicit $\beta$ factor.

In the intermediate and strong coupling regimes (i.e. where the mechanism of polaron dissociation can become relevant), since $\alpha_{2} \gg \omega_{L O}, \operatorname{coth}\left(\beta \alpha_{2} / 2\right) \approx 1$, and we immediately see that the dissociation density does not depend on the temperature:

$$
r_{c}^{(i i)}(T) \approx r_{c}^{(i i)}(T=0)
$$

On the other hand, criterion $(i)$ can be solved to give the critical temperature for crystal melting:

$$
T_{c}=\omega_{\text {ext }} / \ln \left[\left(\sqrt{\frac{r_{s}}{r_{c}^{(i)}(T=0)}}+1\right) /\left(\sqrt{\frac{r_{s}}{r_{c}^{(i)}(T=0)}}-1\right)\right]
$$

At low temperatures, this equation has two different solutions which correspond respectively to the quantum melting analysed in section 4.1, at $r_{s} \approx r_{c}^{(i)}(T=0)$, and to the classical melting due to thermal fluctuations, at $r_{s} \rightarrow \infty$. Let us first expand eq. (42) in the classical case:

$$
T_{c} \simeq \frac{2 R y}{\varepsilon_{s} r_{s}} \delta^{2} \propto n^{1 / 3}
$$

The melting temperature in that case is proportional to the only relevant energy scale in the problem, i.e. the strength of the Coulomb interactions [29]. In the quantum limit, i.e. for $r_{s}$ close to the zero temperature value $r_{c}^{(i)}(T=0), T_{c}$ can be expressed as:

$$
T_{c} \simeq-\omega_{\text {ext }} / \ln \left[\frac{1}{4} \frac{r_{s}-r_{c}^{(i)}(T=0)}{r_{c}^{(i)}(T=0)}\right]
$$

which states that the critical temperature tends to zero at $r_{c}^{(i)}(T=0)$ with a slope that diverges logarithmically. 
In the general case (i.e. at any density and any e-ph coupling), the Lindemann criteria $(i)$ and (ii) can be accurately evaluated as was indicated at the beginning of this section. In figures 8 and 9 , we show two characteristic phase diagrams in the $(T, n)$ plane, which correspond respectively to e-ph coupling constants above and below $\alpha^{*}$. In the strong coupling regime, where $n_{c}^{(i)}>n_{c}^{(i i)}$ (Fig.8,

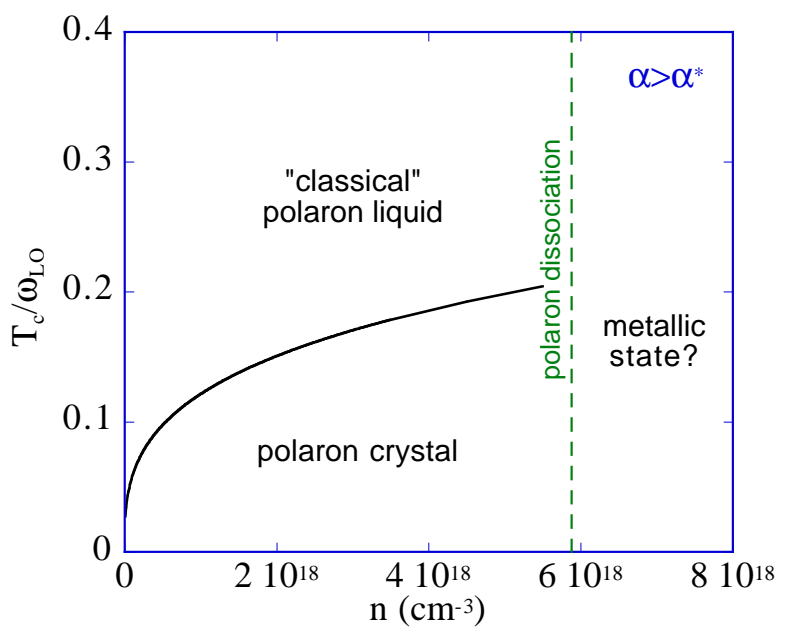

Fig. 8. The $T$ vs. $n$ phase diagram for $\alpha=10>\alpha^{*}$, as obtained from criteria $(i)$ and $(i i)$.

$\alpha=10$ ), while quantum fluctuations lead to polaron dissociation, thermal fluctuations lead to the melting through criterion $(i)$, so that a polaron liquid state can be achieved by increasing the temperature above $T_{c}$. However, a simple argument shows that it is very unlikely that such polaron liquid is degenerate. In fact, the particle statistics become relevant when the de Broglie thermal length is comparable to their average distance, i.e.:

$$
\left(\frac{2 \hbar^{2}}{M_{P} k_{B} T}\right)^{1 / 2} \approx 2 R_{s}
$$

Therefore, the polaron liquid is expected to behave classically down to temperatures of the order

$$
T_{\text {deg }} \approx \frac{m_{e}}{M_{P}} \frac{2 \cdot 10^{5} K}{r_{s}^{2}}
$$

If we consider a polaron mass $M_{P} \approx 10 m_{e}$ and a density $r_{s}=100$, the resulting $T_{\text {deg }}$ is of a few Kelvin, while $T_{c}$ can be as high as some fraction of $\omega_{L O}$ (typically $\sim 100 K$ ). In conclusion, the phase diagram at strong coupling separates into three different regions: polaron crystal (at low density, low temperature), "classical" polaron liquid (at low density, high temperature), and some liquid state at high density where the polarons (or at least some of them) are ionized.

Let us now analyse the situation for $\alpha$ lower than $\alpha^{*}$, where $n_{c}^{(i)}<n_{c}^{(i i)}$ (Fig.9, $\alpha=7.2$ ). In that case, by increasing either the density or the temperature, the crystal melting is always driven by criterion $(i)$. Again, the phase

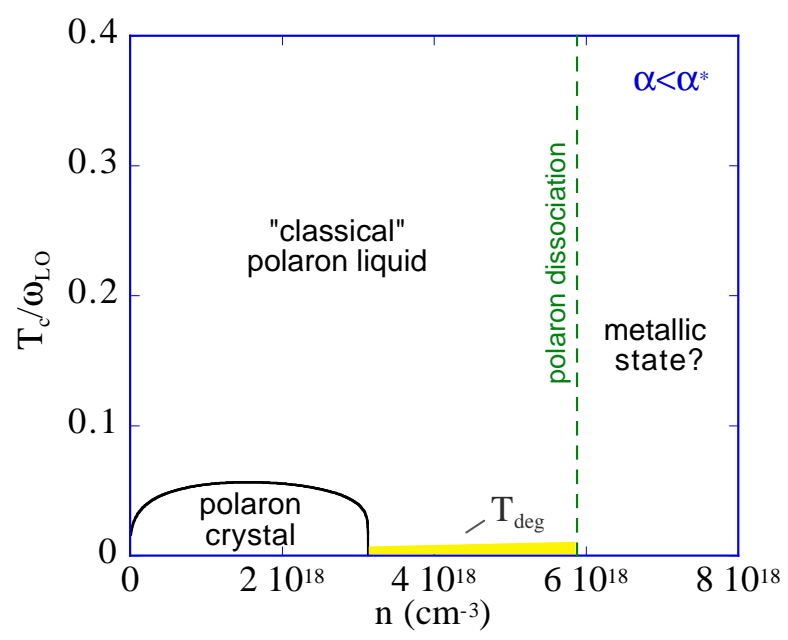

Fig. 9. The $T$ vs. $n$ phase diagram for $\alpha=7.2<\alpha^{*}$, as obtained from criteria $(i)$ and $(i i)$. The shaded area corresponds to temperatures $T<T_{d e g}$, where the polaron liquid could become degenerate.

diagram is divided in three regions, but now the polaron liquid can become degenerate at sufficiently low temperature (i.e. for $T<T_{d e g}$ ), in the intermediate region between $n_{c}^{(i)}$ and $n_{c}^{(i i)}$ (shaded area in Fig.9).

\subsection{Extension to the anisotropic case}

Both the static $\left(\varepsilon_{s}\right)$ and high frequency $\left(\varepsilon_{\infty}\right)$ dielectric constants have been measured in some undopped cuprates. For the parent compound $\mathrm{La}_{2} \mathrm{CuO}_{4}$, it was found [30] $\varepsilon_{s} \approx 30$ and $\varepsilon_{\infty} \approx 5$. Surprisingly, the static dielectric constant remains almost isotropic in the three directions of space (c-axis, and ab-plane of cuprates), which means that the phonons seem to act in the same manner in these three directions. However, it is known that the transport properties are incoherent in the c-direction of $\mathrm{CuO}_{2}$ plane. A possible way to simulate such a specific situation, is to consider a system where the electron bare band mass $m^{*}$ is highly anisotropic, while the Coulomb interactions (and the electron-phonon interaction) remain isotropic. This problem can be studied by considering a crystallized state of Fröhlich polarons, where the localizing potential in the Wigner approximation is spherically symmetric, but the electron effective band masses satisfy $b=m_{x y} / m_{z} \ll 1$.

If we assume $b \rightarrow 0$, which is equivalent to confining the carriers in 2D layers, it is known that the polaron bound states become anisotropic (in this limit, they are usually called "surface" polarons 31]). Correspondingly, the expression (35) for the variational energy at zero temperature must be replaced by:

$$
\begin{aligned}
E & =C_{0}+\left(\alpha_{1}+\alpha_{2}-w\right)-\frac{\left(w^{2}-\alpha_{1}^{2}\right)\left(\alpha_{2}^{2}-w^{2}\right)}{2\left(\alpha_{1}+\alpha_{2}\right) w^{2}} \\
& -\frac{\alpha}{\sqrt{\pi}} \frac{\pi}{2} \int_{0}^{\infty} \frac{e^{-t} d t}{\sqrt{\frac{A_{1}}{\alpha_{1}}\left(1-e^{-\alpha_{1} t}\right)+\frac{A_{2}}{\alpha_{2}}\left(1-e^{-\alpha_{2} t}\right)}}
\end{aligned}
$$


If we compare this expression with (35), we see that apart from a numerical coefficient, the function to be minimized in $2 \mathrm{D}$ is equivalent to the one in the $3 \mathrm{D}$ case, provided that we multiply $\alpha$ by a factor $3 \pi / 4$ (such a scaling relation was first derived by Peeters et al. 32 in the case of an isolated polaron). In our case, it is easy to see that, at a given density, the variational parameters in $2 \mathrm{D}$ are given by the simple relations

$$
\begin{aligned}
& \alpha_{2}^{2 D}(\alpha)=\alpha_{2}^{3 D}(3 \pi \alpha / 4) \\
& \alpha_{1}^{2 D}(\alpha)=\alpha_{1}^{3 D}(3 \pi \alpha / 4)
\end{aligned}
$$

so that the phase diagram in the anisotropic case can be easily deduced from the 3D results (see Fig.77, grey lines [33). The main conclusions are summarized in Table 1, for two different values of the electron band mass. We

\begin{tabular}{l|ccc}
\hline \hline & $\alpha^{*}$ & $n_{c}^{(i i)}\left(m^{*}=m_{e}\right)$ & $n_{c}^{(i i)}\left(m^{*}=2 m_{e}\right)$ \\
\hline isotropic (3D) & 7.5 & $6 \cdot 10^{18}$ & $5 \cdot 10^{19}$ \\
anisotropic (2D) & 3.2 & $8 \cdot 10^{19}$ & $6 \cdot 10^{20}$ \\
\hline \hline
\end{tabular}

Table 1. Critical density $n_{c}^{(i i)}\left(\mathrm{cm}^{-3}\right)$ and coupling $\alpha^{*}$ (see text) 34 .

first observe that the critical coupling above which polaron dissociation becomes effective is strongly reduced from $\alpha^{*}(3 D) \approx 7.5$ (which corresponds to $\omega_{L O} \approx 6 \mathrm{meV}$ if we take $\left.m *=m_{e}\right)$, to $\alpha^{*}(2 D) \approx 3.2\left(\omega_{L O} \approx 40 \mathrm{meV}\right)$. This is reminiscent of the fact that the e-ph coupling has a stronger effect in lower dimensions. Moreover, we see that the critical density $n_{c}^{(i i)}$ is increased: with the parameters of $\mathrm{La}_{2} \mathrm{CuO}_{4}\left(m^{*}=2 m_{e}, \varepsilon_{s}=30, \varepsilon_{\infty}=5\right.$ 30,35), one obtains $n_{c}^{(i i)} \approx 6 \cdot 10^{20} \mathrm{~cm}^{-3}$, in qualitative agreement with experiments. These results suggest that the mechanism of polaron dissociation could be relevant in the description of the IMT in the high- $T_{c}$ cuprates. Note however that it does not mean that the electron motion would become coherent in the c-direction above the dissociation. The resulting state above the dissociation is out of the goal of the present paper.

\section{Conclusion}

In this work, we have attacked the problem of the insulatorto-metal transition in doped polar semiconductors. Neglecting disorder, and replacing the doping ions by a rigid compensating jellium, the two main ingredients in the problem are: (i) the electron-phonon interaction, which leads to polaron formation and (ii) the Coulomb repulsion between the carriers, which causes their crystallization at low density. We have studied the properties of the insulating crystallized state in the framework of Wigner's mean-field approximation. The results show that, upon increasing the Fröhlich e-ph coupling $\alpha$, the Wigner crystal of electrons evolves continuously towards a polaron crystal. On the other hand, a definite distinction between the weak and strong e-ph coupling limits comes from the melting mechanism occurring for increasing density. By applying the Lindemann criterion both to the polaron center-ofmass $\mathbf{R}$ and to the internal coordinate $\mathbf{r}$, we have shown that, at $T=0$, the crystal melting due to the fluctuations of the localized particles is only possible for $\alpha<\alpha^{*}$. In the opposite situation $\alpha>\alpha^{*}$, the IMT is driven by polaron dissociation: the particles become more and more localized due to the increase in their effective mass, and the only way to obtain a metallic state is to ionize the polaron bound states. We have also analysed the melting at finite temperature, and we have proposed an approximate $T$ vs. $n$ phase diagram for the many-polaron problem. Finally, we have shown that if the carriers are confined in 2D layers, the effect of the e-ph interactions is strongly enhanced, so that the mechanism of polaron dissociation can be relevant at rather moderate couplings $(\alpha \approx 3)$.

The overall picture which comes out from this work suggests that large polarons should play an important role in the superconducting cuprates. Moreover, it will be shown in a forthcoming paper 17 by fully taking into account the dipole-dipole interactions between polarons that, due to the peculiar dielectric properties of the PWC, if some itinerant carriers could coexist with localized polarons, the long-range part of their mutual interactions would be overscreened. This would lead to a superconducting instability rather than to an insulator-to-metal transition, as was already pointed out in reference [15].

It is also interesting to compare our results on the melting of a PWC to the studies carried out by De Filippis et al. for the same model, but in the metallic phase [4]. These authors found that at intermediate e-ph coupling ( $\alpha \approx 6)$, the metallic phase can become unstable with respect to the formation of a Charge Density Wave (CDW), as the density is decreased down to $n \approx 10^{18-20} \mathrm{~cm}^{-3}$. For the same value of $\alpha$, we find that in the same range of densities, the PWC becomes unstable with respect to the formation of a polaron liquid. In this sense, both studies on the same problem, either starting from the low density limit or from the high density metallic phase, seem to be quite consistent. Nevertheless, our analysis suggests that the melting of the PWC at zero temperature is first order, as is the case for the Mott transiton (the free carrier density is a discontinuous quantity at the transition). On the contrary, the occurence of a CDW instability should rather be second order (the amplitude of the component $\rho_{q}$ of the electronic density with CDW-wavevector $q$ being the order parameter). We believe that at stronger coupling, where the polaron dissociation holds, there should exist a metastability of the metallic phase with respect to the PWC. This could be related to the fact that the long-range Coulomb interactions (i.e. both the electronelectron and the electron-phonon interactions) are completely unscreened in the crystallized state.

The behaviour of the long-range Coulomb forces being an essential ingredient to the understanding of this "intermediate density" physics is also suggested by a recent work by Leggett 36]. Arguing, on the basis of experimental data on the cuprates, that the Coulomb energy at the 
metal-superconducting transition is saved in the mid infrared range at small $q$ vectors, Leggett has pointed out that the basic pairing mechanism in high-Tc superconductors should also involve small in-plane $q$ vectors, and thus could be due to long-range interactions.

Finally, we would like to mention that the present theoretical work can also find applications in different classes of physical systems. In particular, it was shown by Jackson and Platzman 37 that the dynamics of ripplons coupled to a $2 \mathrm{D}$ electron set on a film of liquid helium can be viewed as a 2D Fröhlich polaron problem.

This work received financial support from the European Commission (contract no. ERBFMBICT 961230).

\section{A Details of the Feynman treatment}

Here we evaluate the upper bound to the free energy $F$ according to eq. (33), that we rewrite here as

$$
F=F_{0}+A+B
$$

\section{A.1 Evaluation of the variational free energy $F$}

After the diagonalization has been carried out, the model (22) represents two independent harmonic oscillators in three dimensions. The corresponding partition function is then

$$
Z_{0}=\left(2 \sinh \beta \alpha_{1} / 2\right)^{-3}\left(2 \sinh \beta \alpha_{2} / 2\right)^{-3}
$$

This result must be equivalent to the path integral of the electronic action (21), including the part corresponding to the motion of the auxiliary particle $\mathbf{X}$. In other words,

$$
Z_{0}=(2 \sinh \beta w / 2)^{-3} \int \mathcal{D}(\text { path }) e^{S_{0}[\mathbf{u}]}
$$

If we equate these two results, and make use of the definition (34), we find:

$$
F_{0}=\frac{3}{\beta} \log \left[\frac{\sinh \beta w / 2}{2 \sinh \beta \alpha_{1} / 2 \sinh \beta \alpha_{2} / 2}\right]
$$

Let us now calculate the term $B=\left\langle S_{0}\right\rangle / \beta$, which is defined as

$$
B=-\frac{C}{2 \beta} \int_{0}^{\beta} \int_{0}^{\beta} d t d s G_{w}(\beta, t-s)\left\langle|\mathbf{u}(t)-\mathbf{u}(s)|^{2}\right\rangle
$$

where $G_{w}(\beta, t-s)$ is the phonon propagator defined in section 2.1, and $C=K w / 4$. The integrand in $B$ can be obtained by expanding up to 2 nd order in $k_{x}$ the expression

$$
I\left(k_{x}, t, s\right)=\left\langle e^{i k_{x}[x(t)-x(s)]}\right\rangle
$$

( $x$ is one of the three equivalent cartesian coordinates: $\mathbf{u}=(x, y, z))$. If we write explicitly the path integral in the last expression and put $f(\tau)=i k_{x} \delta(\tau-t)-i k_{x} \delta(\tau-s)$, eq. (53) takes the form

$$
\begin{aligned}
& I\left(k_{x}, t, s\right) \propto \int \mathcal{D}(\text { path }) \exp \left[-\frac{1}{2} \int \dot{x}^{2} d \tau-\frac{\omega_{W}^{2}}{2 \varepsilon_{s}} \int x^{2} d \tau\right. \\
& -\frac{C}{2} \iint|x(\tau)-x(\sigma)|^{2} G_{w}(\beta, \tau-\sigma) d \tau d \sigma \\
& \left.+\int f(\tau) \cdot x(\tau) d \tau\right]
\end{aligned}
$$

The calculation of such a gaussian integral is standard: first evaluate the function $\bar{x}(\tau)$ for which the exponent is maximum, subject to the boundary condition $\bar{x}(0)=$ $\bar{x}(\beta)=0$, then (within an unimportant constant) the integral (54) reduces to

$$
\begin{aligned}
& I\left(k_{x}, t, s\right)=\exp \left[-\frac{1}{2} \int \dot{\bar{x}}^{2} d \tau-\frac{\omega_{W}^{2}}{2 \varepsilon_{s}} \int \bar{x}^{2} d \tau\right. \\
& -\frac{C}{2} \iint|\bar{x}(\tau)-\bar{x}(\sigma)|^{2} G_{w}(\beta, \tau-\sigma) d \tau d \sigma \\
& \left.+\int f(\tau) \bar{x}(\tau) d \tau\right]
\end{aligned}
$$

which can be simplified to

$$
I\left(k_{x}, t, s\right)=\exp \left\{\frac{1}{2} \int f(\tau) \bar{x}(\tau) d \tau\right\}=e^{\frac{i k_{x}}{2}[\bar{x}(t)-\bar{x}(s)]}
$$

The function $\bar{x}(\tau)$ is the solution of the integral equation

$$
\ddot{x}(\tau)=2 C \int[x(\tau)-x(\sigma)] e^{-w|\tau-\sigma|} d \sigma+\frac{\omega_{W}^{2}}{\varepsilon_{s}} x(\tau)-f(\tau)
$$

which can be solved by defining the auxiliary function

$$
X(\tau)=\frac{w}{2} \int G_{w}(\beta, \tau-\sigma) x(\sigma) d \sigma
$$

Now the differential system reads

$$
\begin{aligned}
\ddot{x}(t) & =\frac{4 C}{w}[x(t)-X(t)]+\frac{\omega_{W}^{2}}{\varepsilon_{s}} x(t)-f(t) \\
\ddot{X}(t) & =w^{2}[X(t)-x(t)]
\end{aligned}
$$

In fact, the problem is equivalent to solving the equations of motion of the two-body model (22) under the external driving force $f(\tau)$. After some lenghty algebra, we find

$$
I\left(k_{x}, t, s\right)=\exp \left\{-\frac{k_{x}^{2}}{2} g(t-s)\right\}
$$

with

$$
g(t-s)=\frac{A_{1}}{\alpha_{1}} g_{1}(t-s)+\frac{A_{2}}{\alpha_{2}} g_{2}(t-s)
$$

and

$$
g_{i}(t-s)=\frac{\cosh \beta \alpha_{i} / 2-\cosh \alpha_{i}(t-s-\beta / 2)}{\sinh \beta \alpha_{i}} ; i=1,2
$$


In the isotropic case, by expanding to second order in $k$ both equations (53) and (59), with $k^{2}=k_{x}^{2}+k_{y}^{2}+k_{z}^{2}$ (the three directions are equivalent) and performing the time integrations in eq. (52) we obtain

$$
B=-\frac{3 C}{w\left(\alpha_{2}^{2}-\alpha_{1}^{2}\right)}\left[\alpha_{2} \operatorname{coth} \frac{\beta \alpha_{2}}{2}-\alpha_{1} \operatorname{coth} \frac{\beta \alpha_{1}}{2}\right]
$$

The term $A=-\langle S\rangle / \beta$ is defined as

$$
A=\frac{\alpha}{\sqrt{8} \beta} \int_{0}^{\beta} \int_{0}^{\beta}\left\langle|\mathbf{u}(t)-\mathbf{u}(s)|^{-1}\right\rangle G_{\omega_{L O}}(\beta, t-s) d t d s
$$

It can be calculated by introducing the Fourier transform

$$
\begin{array}{r}
\left\langle|\mathbf{u}(t)-\mathbf{u}(s)|^{-1}\right\rangle=\int \frac{d^{3} k}{2 \pi^{2} k^{2}}\left\langle e^{i \mathbf{k} \cdot[\mathbf{u}(t)-\mathbf{u}(s)]}\right\rangle \\
=\int \frac{d^{3} k}{2 \pi^{2} k^{2}} I\left(k_{x}, t-s\right) I\left(k_{y}, t-s\right) I\left(k_{z}, t-s\right)
\end{array}
$$

The integration over $k$ gives the result

$$
A=-\frac{\alpha}{\sqrt{\pi}} \frac{1}{1-e^{-\beta}} \int_{0}^{\beta} \frac{d t e^{-t}}{\sqrt{g(t)}}
$$

If we take the limit $\beta \rightarrow \infty$, and include the constant part $C_{0}=-\left(9 \tilde{\varepsilon}^{2} \alpha^{2} / 5 \varepsilon_{s} r_{s}\right)\left(m_{e} / m^{*}\right)$, the upper bound to the ground-state energy takes the form

$$
\begin{aligned}
E & =C_{0}+\frac{3}{2}\left(\alpha_{1}+\alpha_{2}-w\right)-\frac{3\left(w^{2}-\alpha_{1}^{2}\right)\left(\alpha_{2}^{2}-w^{2}\right)}{4\left(\alpha_{1}+\alpha_{2}\right) w^{2}} \\
& -\frac{\alpha}{\sqrt{\pi}} \int_{0}^{\infty} \frac{e^{-t} d t}{\sqrt{\frac{A_{1}}{\alpha_{1}}\left(1-e^{-\alpha_{1} t}\right)+\frac{A_{2}}{\alpha_{2}}\left(1-e^{-\alpha_{2} t}\right)}}
\end{aligned}
$$

\section{A.2 Evaluation of the internal and external fluctuations}

Here we calculate the average displacements of the centerof-mass and of the relative coordinate, which enter in the Lindemann criteria $(i)$ and $(i i)$. In each space direction, since the eigenmodes are independent, we have $\left\langle u_{1} u_{2}\right\rangle=$ $\left\langle u_{1}\right\rangle\left\langle u_{2}\right\rangle$ so that making use of eq. 27) and (30), we get the dimentionless quantities

$$
\begin{aligned}
& \frac{\left\langle\delta R^{2}\right\rangle}{\left(2 m^{*} \omega_{L O}\right)^{-1}}=\left(\frac{m^{*}}{M_{P}}\right)^{2}\left[A_{1}^{2} \frac{\alpha_{2}^{4}}{w^{4}}\left\langle\delta u_{1}^{2}\right\rangle+A_{2}^{2} \frac{\alpha_{1}^{4}}{w^{4}}\left\langle\delta u_{2}^{2}\right\rangle\right] \\
& \frac{\left\langle\delta r^{2}\right\rangle}{\left(2 m^{*} \omega_{L O}\right)^{-1}}=\left(\frac{\alpha_{1}^{2}}{\alpha_{2}^{2}-\alpha_{1}^{2}}\right)^{2}\left\langle\delta u_{1}^{2}\right\rangle+\left(\frac{\alpha_{2}^{2}}{\alpha_{2}^{2}-\alpha_{1}^{2}}\right)^{2}\left\langle\delta u_{2}^{2}\right\rangle
\end{aligned}
$$

where

$$
\begin{aligned}
& \left\langle\delta u_{1}^{2}\right\rangle=\left(2 A_{1} \alpha_{1}\right)^{-1} \operatorname{coth} \frac{\beta \alpha_{1}}{2} \\
& \left\langle\delta u_{2}^{2}\right\rangle=\left(2 A_{2} \alpha_{2}\right)^{-1} \operatorname{coth} \frac{\beta \alpha_{2}}{2}
\end{aligned}
$$

are the r.m.s. displacements of two harmonic oscillators at finite temperature. Observing that the polaronic length unit is $\left(2 m^{*} \omega_{L O}\right)^{-1 / 2}=\left(m / m^{*}\right) \tilde{\varepsilon} \alpha a_{0}$, we can write

$$
\begin{aligned}
\frac{\sqrt{\left\langle\delta R^{2}\right\rangle}}{R_{s}} & =\left(\frac{\omega_{W}^{2}}{4 \alpha}\right)^{1 / 3} \frac{m^{*}}{M_{P}} \times \\
& \times \sqrt{\frac{A_{1}}{\alpha_{1}}\left(\frac{\alpha_{2}}{w}\right)^{4} \operatorname{coth} \frac{\beta \alpha_{1}}{2}+\frac{A_{2}}{\alpha_{2}}\left(\frac{\alpha_{1}}{w}\right)^{4} \operatorname{coth} \frac{\beta \alpha_{2}}{2}} \\
\frac{\sqrt{\left\langle\delta r^{2}\right\rangle}}{R_{s}} & =\left(\frac{\omega_{W}^{2}}{4 \alpha}\right)^{1 / 3} \frac{1}{\alpha_{2}^{2}-\alpha_{1}^{2}} \times \\
& \times \sqrt{\frac{\alpha_{1}^{3}}{A_{1}} \operatorname{coth} \frac{\beta \alpha_{1}}{2}+\frac{\alpha_{2}^{2}}{A_{2}} \operatorname{coth} \frac{\beta \alpha_{2}}{2}}
\end{aligned}
$$

\section{References}

1. A.S. Alexandrov and N.F. Mott, Rep. Prog. Phys. 57, 1197 (1994)

2. G. Mahan, in Polarons in Ionic Crystals and Polar semiconductors, ed. J. Devreese (1972)

3. L.F. Lemmens, J.T. Devreese and F. Brosens, Phys. Stat. Sol. B 82, 439 (1997)

4. G. de Filippis, V. Cataudella and G. Iadonisi, Eur. Phys. J. B 8, 339 (1999); G. Iadonisi, G. Capone, V. Cataudella, G. de Filippis, Phys. Rev. B 53, 13497 (1996)

5. E. Wigner, Phys. Rev., 46, 1002 (1934)

6. P. Quémerais, Mod. Phys. Lett. B 9, 25, 1665 (1995)

7. A.A. Remova, B. Ya. Shapiro, Physica C 160, 202 (1989)

8. We call "external" any doping charge added to the host material.

9. L. D. Landau, Phys. Z. Sowjetunion, 3, 664 (1933)

10. H. Frohlich, Adv. Phys. 3, 325 (1954)

11. V. L. Vinetskii and M. S. Giterman, Soviet. Phys. JETP, 6, 1796 (1958); G. Verbist, F. M. Peeters, and J. T. Devreese, Phys. Rev. B 43, 2712 (1991); F. Bassani, M. Geddo, G. Iadonisi and D. Ninno, Phys. Rev. B 43, 5296 (1991)

12. G.R. Allcock, Adv. Phys. 5, 412 (1956)

13. N. F. Mott, Phil. Mag. 6, 2897 (1961)

14. P. Quémerais and S. Fratini, Mod. Phys. Lett. B 11, 30, 1303 (1997)

15. S. Fratini and P. Quémerais, Mod. Phys. Lett. B 12, 24, 1003 (1998); P.Quémerais and S. Fratini, Int. Journ. Mod. Phys. B 12, 3131 (1998)

16. S. Fratini, PhD dissertation, Grenoble 1999, unpublished

17. S. Fratini and P. Quémerais, in preparation

18. R. P. Feynman, Phys. Rev. 97, 3, 660 (1955); R.P. Feynman and A.R. Hibbs, Quantum Mechanics and Path Integrals, Mc Graw-Hill, New York (1965)

19. P. Nozières and D. Pines, Phys. Rev., 111, 2, 442 (1958)

20. W.J. Carr, Phys. Rev 122, 1437 (1961)

21. In refs. 6, 14, 15, the notation $\omega_{\text {ext }}, \omega_{\text {int }}$ was used instead of $\alpha_{1}, \alpha_{2}$ for the eigenfrequencies of the two-body model.

22. B. Gerlach and H. Löwen, Rev. Mod. Phys. 63, 63 (1991)

23. E. Kartheuser, Polarons in Ionic Crystals and Polar Semiconductors, North Holland, Amsterdam (1972), p.515-550; D. Emin, Phys. Rev. B 48 (1993), p. 13691; A.E. Myasnikova and E.N. Myasnikov, Phys. Rev. B 565316 (1997)

24. C.M. Care, and N.H. March, Adv. Phys. 24, 101 (1975) 
25. D.M. Ceperley and B.J. Alder, Phys. Rev. Lett. 45, 566 (1980); M.D. Jones and D.M. Ceperley, Phys. Rev. Lett. 76, $4572(1996)$

26. T. D. Schultz, Phys. Rev. 116, 3, 526 (1959)

27. A. Bianconi, Proceed. of the Int. Conf. on Materials and mechanism of Superconductivity High Temperature Superconductors IV, Grenoble (1994), ed. P. Wyder (North-Holland), p. 269, and ref. therein; D. Mihailovic and K.A. Müller, Proc. NATO ASI "Materials aspects of High-Tc Superconductivity: 10 years after the discovery", Kluwer, London (1997), p.243 ; K.A. Muller, G. Zhao, K. Conder, H. Keller, J.Phys.Cond.Mat., 10, L291 (1998)

28. M.A. Krivoglatz and S.I. Pekar, Izv. Acad. Nauk. SSSR 21, 1 (1957); Y. Osaka, Progr. Theor. Phys. 22, 437 (1959); D.P.L. Castrignano et al., Phys. Lett. 104A, 364 (1984)

29. The correct value of $\delta$ for classical melting is 0.155 , as can be deduced from MC simulations 25.

30. C.Y. Chen et al., Phys. Rev. B. 43, 392 (1991)

31. J. Sak, Phys. Rev. B 6, 3981 (1972)

32. F. Peeters and J.T. Devreese, Phys. Rev. B 36, 4442 (1987)

33. In the limit of vanishing e-ph coupling, the melting density appears to be independent on the dimensionality. This is due to the fact that we have considered the same value of $\delta=0.25$ both in the $3 \mathrm{D}$ and in the $2 \mathrm{D}$ case. As a consequence, the critical density estimated in the anisotropic case should be considered as a lower bound.

34. In previous references, the value $\alpha^{*}=4.4$ that we referred to as "fully anisotropic", was in fact obtained by taking a small but finite value $b \approx 0.01$.

35. C.G. Olson et al., Phys. Rev. B 42, 381 (1990); Y.J. Uemura, Phys. Rev. Lett. 62, 2317 (1989)

36. A. J. Leggett, Phys. Rev. Lett. 83, 392 (1999); J. Phys. Chem. Solids 59, 1729 (1998)

37. S. A. Jackson and P. M. Platzman, Surf. Science 113, 401 (1982) 